\title{
IMPLEMENTACIÓN Y EVALUACIÓN DE UN SISTEMA SILVOPASTORIL EN EL CHOCÓ BIOGEOGRÁFICO COLOMBIANO
}

\author{
Implementation and evaluation of a silvopastoral system in the Colombian \\ biogeographic Chocó
}

Artículo de Investigación

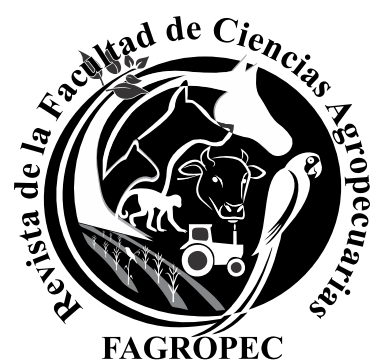

Recibido 21 de agosto de 2019. Aceptado 29 de noviembre de 2019.

${ }^{1}$ Zootecnista, M. Sc. Ph. D. Profesor Asociado tiempo completo, Universidad de Nariño. Pasto. Colombia , Grupo de Investigación Producción y Sanidad Animal - Cuyes, Linea de Investigación: Recursos Alimentarios para Especies Pecuarias.

(iD https://orcid.org/0000-0003-4454-3405

${ }^{2}$ Zootecnista, M. Sc. Ph. D. Profesor Asociado tiempo completo, Universidad de Nariño. Pasto. Colombia, Grupo de Investigación Producción y Sanidad Animal - Cuyes, Linea de Investigación: Recursos Alimentarios para Especies Pecuarias.

(iD https://orcid.org/0000-0002-8161-8229

${ }^{3}$ Ing. Agrónomo, M.Sc. Profesor hora catedra. Universidad de Nariño. Pasto. Colombia, Grupo de Investigación en Recursos Naturales y Agroforestería, Linea Fitomejoramiento.

(iD) https://orcid.org/0000-0001-7348-0912

${ }^{4}$ Zootecnista, M.Sc. Profesor Titular tiempo completo Universidad de la Amazonia. Florencia. Caquetá. Colombia, Grupo de

*Autor para Correspondencia: galvezceron@hotmail.com
Arturo Leonel Gálvez Cerón ${ }^{1 *}$, José Edmundo Apráez Guerrero², José Julian Apráez Muñoz ${ }^{3}$, Fredy Rodolfo Ruales España ${ }^{4}$

\section{RESUMEN}

Se implementó un sistema silvopastoril multiestrato (SSPm) en una zona de bosque húmedo tropical (bh-T), en el Municipio de Tumaco, Departamento de Nariño, Colombia, para medir su impacto en la diversidad florística en los sistemas ganaderos, la mesofauna edáfica en el Sistema Silvopastoril y la producción y calidad de la oferta alimentaria, comparado con un sistema convencional (SC) basado en pastizales. Adicionalmente, se realizó una caracterización del proceso de regeneración natural en el Sistema Silvopastoril, a través de análisis de estructura horizontal, abundancia de especies, dominancias absolutas y frecuencia. La composición y diversidad florística se determinó mediante los índices de Shannon-Wiener, Margalef y de Simpson. Los arreglos silvopastoriles se evaluaron con un diseño experimental en bloques completos al azar con arreglo en parcelas divididas. El análisis del suelo reportó una textura arcilloso-arenoso, relativamente pesado, con una densidad aparente de 1,04 y 1,12 g cm3 en el Sistema Silvopastoril y convencional respectivamente. Los contenidos de $\mathrm{N}$ $(0,07 \%), \mathrm{K}(0,43 \mathrm{cmol} \mathrm{kg}-1)$ y $\mathrm{MO}(2,04 \%)$ resultaron mayores en el SSPm, hubo mayor presencia de individuos descomponedores de materia orgánica, como lombrices, en el Sistema Silvopastoril, en ambas épocas. Se evidenció cómo los sistemas silvopastoriles favorecen la producción de biomasa comestible (17,82 del SSPm frente a 11,97 Ton MS ha-1 año-1 en el SC). Se encontró un total de 38 especies entre herbáceas, arbustivas y arbóreas, pertenecientes a 25 familias y 35 géneros, siendo las familias más representativas Fabaceae, Urticaceae y Solanaceae. Se puede concluir que este tipo de sistemas representa una alternativa favorable para la ganadería en región húmeda.

Palabras claves:

Biomasa comestible; bromatológico; macrofauna; metabolitos secundarios; regeneración natural asistida.

\section{ABSTRACT}

Was implemented a multi-layer Silvopastoral system (mlSPS) in an area of Tropical humid forest in the municipality of Tumaco, Department of Nariño, to measure its impact on the diversity of flora in livestock systems, the edaphic mesofauna, and the production and quality of the food supply, 
REVISTA FAGROPEC

UNIVERSIDAD DE LA AMAZONIA - FLORENCIA-CAQUETA
Implementación y evaluación de un sistema silvopastoril en el Chocó
Investigación en Producción y Salud AnimalGIPSA- Linea de Investigación en Sistemas Silvopastoriles.

https://orcid.org/0000-0002-3494-4398

\section{Como citar:}

GÁLVEZ CERÓN, Arturo Leonel, et al. Implementación y Evaluación de un Sistema Silvopastoril en el Chocó biogeográfico colombiano. En: Revista Facultad Ciencias Agropecuarias - FAGROPEC. Universidad de la Amazonia, Florencia - Caquetá. Volumen 12 enero-junio, 2020. Pp. 129-142 ISSN-Revista en Línea: 2539-178X compared to conventional system (CS), based grass monoculture. In addition, a characterization of the natural regeneration process was performed in the silvopastoral system, through horizontal structure analysis, abundance of species, absolute dominance and frequency. The composition and floristic diversity it was determined by the indices of Shanon-Wiener and of Simpson. Soil analysis showed relatively heavy clay-sandy soils, with an apparent density of 1.04 and $1.12 \mathrm{~g} \mathrm{~cm}-3$ in the Silvopastoral System and conventional respectively. The levels of $\mathrm{N}(0.07 \%), \mathrm{K}(0.43 \mathrm{cmol} \mathrm{kg}-1)$ and organic matter $(2.04 \%)$ were higher in the mlSPS. There were more OM decomposer individuals, such as worms in the Silvopastoral System in both seasons. It was evidenced that the silvopastoral systems increase the production of edible biomass (17.82 of the mISPS compared to $11.97 \mathrm{Tn}$ DM ha-1 year-1 in the CS). A total of 38 herbaceous, shrub and arboreal species were found, belonging to 25 families and 35 genera; the most representative families were: Fabaceae, Urticaceae and Solanaceae. It is concluded that multi-stratum silvopastoral systems represent a good alternative for tropical humid forest livestock.

Key words:

eatable biomass; bromatologic; Chocó biogeographic; edaphic macrofauna; natural assisted regeneration; secondary metabolites.

\section{INTRODUCCIÓN}

La ganadería bovina de carne ha tenido históricamente un alto costo ambiental en el territorio colombiano. Pérdida de hábitats naturales, fragmentación de ecosistemas y disminución en la productividad de los suelos se cuentan dentro de las consecuencias del modelo ganadero que actualmente prospera en el país (Rico, 2017). Frente a esto, los Sistemas Silvopastoriles (SSP) se presentan como una alternativa que proporciona diversos servicios que ayudan a los ecosistemas, a los productores y a las comunidades a mejorar la producción, al tiempo que dinamizan su adaptación a la variabilidad y el cambio climático (Pérez-Almario et al. 2017). Además, estos bienes y servicios proporcionados por los SSP pueden considerarse más importantes para los ecosistemas, generando ventajas en el uso y en las funciones no sólo a nivel de especies sino a nivel del ecosistema en conjunto (Ospina et al.2016).

La Granja Maragrícola de la Universidad de Nariño, ubicada en el Sur Occidente colombiano, desarrolla un programa de producción extensiva para bovinos de carne en terrenos transformados inicialmente en piscinas destinadas a la producción camaronera, función que desempeñó la granja hasta el momento que se donó a la Universidad. Los parámetros productivos en esta zona son bajos (p.e. ganancia de peso de $380 \mathrm{~g}$ animal $^{-1} \mathrm{dia}^{-1}$ ). La implementación de los SSP por regeneración natural asistida diversifica la oferta forrajera, incrementan la calidad nutricional de la dieta $\mathrm{y}$, al mismo tiempo, aseguran la sostenibilidad y el equilibrio ambiental con el uso de diferentes recursos locales (Gálvez-Cerón et al. 2014). En las selvas húmedas mexicanas, los sistemas agroforestales y, particularmente, los sistemas agrosilvopastoriles han contribuido a la conservación de la diversidad, la conservación del suelo y a aumentar la cantidad de alimento tanto para los animales como para las familias (Maya-Martínez et al. 2019). 
En los sistemas pastoriles resulta favorable la siembra de plantas perennes forrajeras, debido a que, en general, mantienen la calidad del suelo (propiedades químicas, físicas y biológicas) debido a la estabilidad en la cobertura vegetal y a la asociación de gramíneas y leguminosas (Hernández-Vigoa et al.2018).

Entre las características que se debe buscar en las especies con potencial forrajero está la cantidad y calidad de la biomasa comestible, la rápida recuperación de rebrotes después del aprovechamiento (Pérez-Almario et al. 2017) y la adaptación a las condiciones edafo-climáticas locales (GálvezCerón et al.2018).

Teniendo en cuenta las anteriores consideraciones, la presente investigación tuvo como finalidad evaluar la mejora en la oferta alimentaria de un sistema silvopastoril multiestrato a partir de la regeneración natural asistida frente a un pastizal natural en una área degradada por remoción del suelo cultivable.

\section{MATERIALES Y MÉTODOS}

\section{Localización}

Este trabajo se desarrolló en la Granja Maragrícola de la Universidad de Nariño, ubicada en la vereda Inguapí Chiricana, km 22 del municipio de Tumaco, con precipitación de $4.000 \mathrm{~mm}$ año ${ }^{-1}$ y temperatura promedio anual de $27^{\circ} \mathrm{C}$, zona de vida de bosque húmedo tropical (bh-T), según Holdridge (1978).

\section{Variables evaluadas}

En un sistema silvopastoril multiestrato (SSPm) y convencional (SC) se evaluaron algunas variables del suelo, como propiedades físicas (densidad aparente), químicas (N-P-K, MO, pH), biológicas (macrofauna edáfica) y productivas (biomasa comestible), en épocas de lluvia y seca. Los análisis físicos, químicos y bromatológicos se realizaron en los Laboratorios Especializados de la Universidad de Nariño.

\section{Variables productivas}

La producción de forraje se realizó mediante aforos en zig-zag utilizando un marco de 1 m de lado (Gálvez-Cerón, 2015). Cada submuestra se pesó con una balanza analítica, luego se tomó el promedio aritmético en $\mathrm{kg} \mathrm{m}^{2}$ en cada estrato, así: Estrato herbáceo: producción de forrajes $\mathrm{m}^{2}$ según método descrito anteriormente. Estrato arbustivo: producción de biomasa comestible (hoja + tallos tiernos) x No. de árboles ha ${ }^{-1}$ (Gálvez-Cerón et al. 2014).

La caracterización del proceso de regeneración se hizo según metodología reportada por Dubois (1980), citado por Melo y Vargas (2003), con la siguiente división de categorías:

$\checkmark \cdot$ Renuevos o brinzales (R): todos los individuos de las especies arbóreas entre 0 y $30 \mathrm{~cm}$ de altura.

$\checkmark \cdot$ Plantones (U1): alturas entre $30-150 \mathrm{~cm}$.

$\checkmark \cdot$ Plantones (U2): alturas entre $150 \mathrm{~cm}$ y $300 \mathrm{~cm}$.

$\checkmark \cdot$ Establecidos (E): alturas superiores a $300 \mathrm{~cm}$ e inferiores a $5 \mathrm{~cm}$ de diámetro normal.

$\checkmark \cdot$ Latizales (L): diámetros entre 5 y $10 \mathrm{~cm}$. 
$\checkmark \cdot$ Fustales o árboles propiamente dichos: por encima de la categoría de los latizales.

Para la elaboración del inventario, la categoría R se evaluó en parcelas de $2 \times 2 \mathrm{~m}$, las categorías U y E se registraron en parcelas de $5 \times 5 \mathrm{~m}$, la categoría L en parcelas de $10 \times 10 \mathrm{~m}$ y, por último, la categoría de fustales se evaluó en toda el área de estudio, $5000 \mathrm{~m}^{2}$.

Para determinar el diámetro normal se utilizó la cinta métrica, medido a 1.30 metros sobre el nivel del suelo (Perla y Torres 2008), y para determinar la altura total del árbol se utilizó una vara de $2 \mathrm{~m}$.

Para determinar el área basal se empleó la siguiente fórmula:

(1) Areabasal $(A B)=\left(\pi^{*}(d)^{2}\right) / 4$

Donde:

$\mathrm{d}=$ diámetro

La clasificación de las especies vegetales se hizo según el Método APG III, en el Herbario PSO de la Universidad de Nariño, Pasto, Colombia, determinando familia, género y especie.

La diversidad y riqueza florística se determinó mediante los índices Shannon-Wiener, Margalef y de Simpson (Moreno 2001, Villareal et al. 2006).

Se hicieron labores de raleo y manejo de especies nativas promisorias ya establecidas en las áreas de estudio, correspondiente a antiguas piscinas para la producción camaronera. Para el enriquecimiento del sistema productivo se procedió a la siembra de botón de oro (Tithonia diversifolia(Hemsl) Gray), guama (Inga sp. Mart.) y martingalvis (Cassia reticulata Willd), presentes en la zona. Se realizó una poda de formación cada seis meses, a una altura de 1,50 m.

\section{Variables bromatológicas}

Se determinó la composición nutricional de las especies comestibles en ambos sistemas (Tithonia diversofolia y Paspalum sp.), de acuerdo con los métodos establecidos por la A.O.A.C. (1995), que incluyen el contenido de humedad (método 930.04), proteína cruda por el método de Kjeldahl (Nx6.25) (método 955.04), cenizas (por calcinación a $550{ }^{\circ} \mathrm{C}$ ) (método 930.05), extracto etéreo (método 962.09) y fibra bruta (método 920.39). Se determinó además metabolitos secundarios como fenoles, esteroides, alcaloides y saponinas a través de la evaluación cualitativa.

\section{Variables biológicas}

La macrofauna edáfica en campo se muestreó según la metodología del programa internacional «Biología y fertilidad del suelo tropical» o TSBF (Anderson e Ingram, 1993), que consistió en la revisión in situ de cuatro monolitos de suelo de $25 \times 25$ × $30 \mathrm{~cm}$ por cada sistema evaluado, con clasificación hasta orden, en ambos sistemas en dos épocas (lluvia y seca), en talud y piso de las antiguas piscinas camaroneras, con apoyo del Laboratorio de Entomología de la Universidad de Nariño.

\section{Análisis estadístico}

Se realizó un diseño experimental de parcelas divididas, en donde los lotes se dividieron en subparcelas (tratamientos) y se tomaron muestras aleatoriamente, cada 30 días durante 4 meses, a 
una profundidad de 20-40 cm, donde se desarrolla el 90\% del sistema radicular de las pasturas (Pérez et al. 2006). Para las pruebas físicas se utilizó la técnica de los cilindros graduados de Chapingo (García 2003).

El trabajo se orientó bajo un diseño experimental en bloques completos al azar con arreglo en parcelas divididas. El arreglo de los tratamientos uno y dos fue factorial con tres factores o estratos (bajo, medio y alto), y un testigo compuesto por una pastura convencional, como lo describe la Tabla 1. Cada tratamiento constó de tres réplicas y cinco repeticiones. Se realizó un Análisis de Varianza (ANDEVA) y una prueba de $\mathrm{T}$ de student $(\mathrm{P}<0,01)$ en el programa estadístico SAS.

Modelo estadístico: $Y i j k=\mu+\alpha i+P k+d i k+\beta j+(\alpha \beta) i j+\varepsilon i j k$

Donde:

Yijk $=$ valor del i-esimo nivel del factor A, j-esimo nivel del factor B, y k-esimo bloque (repetición).

$\mu=$ media general.

$\alpha i=$ efecto del i-esimo nivel del factor A.

$P k=$ efecto del k-esimo bloque.

$d i k=$ error aleatorio de la parcela completa (error1)

$\beta j=$ efecto del i-esimo nivel del factor $\mathrm{B}$.

$(\alpha \beta) i j=$ efecto de interaccion entre ambos factores.

$\varepsilon i j k=$ error aleatorio de la subparcela (Error 2)

Tabla 1. Diseño de los tratamientos y estratos utilizados en la investigación.

\begin{tabular}{cl}
\hline Tratamientos & \multicolumn{1}{c}{ Descripción } \\
\hline T0 & Pastizal convencional: grama natural y arvenses \\
& Estrato bajo: Pastizal convencional \\
& Estrato medio: T. diversifolia. Densidad: $1.000 \mathrm{arb} \mathrm{ha}^{-1}$. \\
& Estrato alto: Inga sp. Densidad: $46 \mathrm{arb} \mathrm{ha}^{-1}$. \\
& Estrato bajo: Pastizal convencional. \\
& Estrato medio: T. diversifolia. Densidad: $1.000 \mathrm{arb} \mathrm{ha}^{-1}$. \\
& Estrato alto: C. reticulata. Densidad: $77 \mathrm{arb} \mathrm{ha}^{-1}$. \\
\hline
\end{tabular}

\section{RESULTADOS Y DISCUSIÓN}

\section{Propiedades físicas del suelo}

La densidad aparente tuvo valores de 1,04 y $1,12 \mathrm{~g} \mathrm{~cm}^{-3}$ en el sistema silvopastoril y convencional respectivamente. Estos resultados son mejores a los encontrados por Hernández-Vigoa et al. (2018) en potrero compuesto por Megathyrsus maximus Jacq., Cynodon nlemfuensis Vanderhyst y Teramnusuncinatus (L.) Sw., y pastizal de Pennisetum sp. y Leucaena leucocephala (Lam.) en la Provincia de Artemisa, Cuba, en época de lluvias, cuyos valores fueron de 1,28 y $1,3 \mathrm{~g} \mathrm{~cm}^{-3}$ respectivamente. La densidad aparente resulta en uno de los indicadores edáficos más importantes para determinar la calidad de un suelo, específicamente en su componente físico (Hernández-Vigoa et al.2018). 
Propiedades químicas del suelo

La Tabla 2 resume los resultados de algunas propiedades químicas evaluadas en los diferentes sistemas.

Tabla 2. Propiedades químicas del suelo.

\begin{tabular}{cccccc}
\hline Sistema & MO (\%) & N (\%) & $\mathbf{P}\left(\mathbf{m g ~ k g} \mathbf{~}^{-\mathbf{1}}\right)$ & $\begin{array}{c}\mathbf{K} \\
\left(\mathbf{c m o l}^{+} \mathbf{~ k g}^{\mathbf{1}}\right)\end{array}$ & $\mathbf{p H}$ \\
\hline SSP & 2,04 & 0,07 & 18,2 & 0,43 & 5,62 \\
SC & 1,7 & 0,06 & 11,8 & 0,28 & 5,66 \\
\hline
\end{tabular}

SSP: Sistema Silvopastoril; SC: sistema convencional; MO: materia orgánica; N: Nitrógeno; P: Fósforo; K: Potasio.

El contenido de materia orgánica fue de $2.04 \%$ en el sistema silvopastoril y $1.7 \%$ en el sistema convencional. El nitrógeno presentó niveles de 0.07 y $0.06 \%$ en el sistema silvopastoril y convencional respectivamente. En pasturas con árboles, la sombra y biomasa de los árboles mejora la fertilidad del suelo, aumenta la disponibilidad de nitrógeno para las especies forrajeras herbáceas y además mejora la calidad del forraje, contribuyendo, incluso, a aumentar la producción de biomasa. Por otro lado, la asociación de gramíneas y árboles forrajeros en sistemas silvopastoriles es una posibilidad para mejorar la disponibilidad de forraje durante el año y, al mismo tiempo, mejorar su calidad químico nutricional (Gaviria et al. 2015).

En el sistema silvopastoril se encontró $18,2 \mathrm{mg} \mathrm{kg}^{-1}$ de fósforo disponible, mientras que en el sistema convencional $11,8 \mathrm{mg} \mathrm{kg}^{-1}$, valor considerado bajo de acuerdo con los estándares establecidos por los Laboratorios Especializados de la Universidad de Nariño. Sin embargo, se observó un contenido mayor en el sistema silvopastoril. El potasio intercambiable $\left(\mathrm{K}^{+}\right)$tuvo un valor medio en el sistema convencional $\left(0,28 \mathrm{cmol}^{+} \mathrm{kg}^{-1}\right)$ y alto en el sistema silvopastoril $\left(0,43 \mathrm{cmol}^{+} \mathrm{kg}^{-1}\right)$. En estos sistemas silvopastoriles se integran una variedad de especies vegetales, entre ellas gramíneas, especies arbustivas y arbóreas, las cuales son capaces de aportar alimentos y bienestar para el ganado, pero, además, generan aportes significativos de materia orgánica al suelo, a través de la acumulación de hojarasca, tallos y producción de biomasa de raíces finas (Vallejo et al. 2012, Martínez et al. 2014).

Aunque la variación entre el pH encontrado en el sistema silvopastoril y el convencional fue mínima (5,62 y 5,66 respectivamente), se debe resaltar que, en presencia de árboles, el pH edáfico tiende a acercarse a la neutralidad. Martínez (2013) encontró niveles de $\mathrm{pH}$ de 5,5 para un cultivo de pasto Dichanthium aristatum y de 6,4 para un arreglo silvopastoril, lo que refiere que los árboles ayudan a neutralizar el $\mathrm{pH}$, puesto que realizan adición de bases al suelo por medio de la hojarasca (Linares 2006). En previos reportes, Stefano y Jacobson (2017) y Priano et al. (2017) reportan que los sistemas que asocian diferente tipo de especies arbóreas en una misma unidad de terreno poseen mayor capacidad de acumulación de nutrientes en el suelo que un sistema sin árboles basado sólo en las pasturas, mejorando no sólo las propiedades físicas si no también químicas en el suelo, como se demuestra en los resultados de esta investigación, en donde, al comparar los tratamientos, se puede observar que dichas propiedades presentan valores mayores en las variables del suelo al comparar un SSP con un sistema convencional de pasturas, como lo muestra la Tabla 2. 
Propiedades biológicas del suelo

En el Tabla 3 se muestra la clasificación en orden y familia de los individuos encontrados en el sistema silvopastoril y el convencional, en las dos épocas.

Tabla 3. Macrofauna edáfica.

\begin{tabular}{|c|c|c|c|c|c|c|c|}
\hline \multirow[t]{3}{*}{ Orden } & \multirow[t]{3}{*}{ Familia } & \multicolumn{4}{|c|}{ Sistema Silvopastoril } & \multirow{2}{*}{\multicolumn{2}{|c|}{ S. Convencional }} \\
\hline & & \multicolumn{2}{|c|}{ E.S. } & \multicolumn{2}{|c|}{ E.L. } & & \\
\hline & & $\mathrm{T}$ & $\mathrm{P}$ & $\mathrm{T}$ & $\mathrm{P}$ & $\mathrm{T}$ & $\mathrm{P}$ \\
\hline \multirow[t]{2}{*}{ Coleópteros } & Escarabidae & 16 & - & 80 & 16 & - & - \\
\hline & Passalidae & - & 16 & - & - & - & - \\
\hline Himenópteros & Formicidae & 928 & 240 & 1104 & 384 & 464 & 400 \\
\hline \multirow{2}{*}{ Lepidópteros } & Geometridae & 16 & - & 16 & - & - & - \\
\hline & Nymphalidae & 32 & - & 16 & - & - & - \\
\hline Hemípteros & Pentatomidae & 16 & - & 64 & 16 & - & - \\
\hline Orthopteros & Acrididae & 32 & - & - & - & - & - \\
\hline Miriápodos & & 624 & 128 & 832 & 240 & - & 144 \\
\hline Haplotaxida & Lombricidae & 2256 & 560 & 4448 & 1392 & 192 & 304 \\
\hline Blattodea & & . & - & - & - & - & 32 \\
\hline Isopoda & & - & - & - & - & 80 & 320 \\
\hline \multirow[t]{2}{*}{ Araneae } & & - & - & - & - & - & 32 \\
\hline & Total & 3920 & 944 & 6560 & 2048 & 736 & 1232 \\
\hline
\end{tabular}

E.S.: Época seca; E.L.: Época de lluvias; T: Talud; P: Piso

En el sistema silvopastoril se encontró gran cantidad de individuos descomponedores de materia orgánica, familia Lombricidae, y un bajo número de organismos defoliadores, como las familias Geometridae y Nymphalidae del orden Lepidóptera; de hecho, se evidenció un incremento entre una época y otra, destacándose la época de lluvias como refugio para diversidad de especies.

Reina y Meneses (2015) encontraron mayor diversidad y riqueza de macrofauna edáfica en un sistema de pastizal (Panicum maximum, Brachiaria decumbens e Hyparrhenia rufa) con árboles dispersos de Senna spectabilis respecto al sistema convencional de pastizal solo, en una zona de bs-T en el Departamento de Nariño, Colombia, debido muy seguramente a las mejores condiciones de temperatura, humedad y aporte de hojarasca del sistema arborizado.

\section{Diversidadflorística}

Los índices de diversidad (Simpson y Shannon), riqueza (Margaleff) y dominancia (Simpson) se muestran en la Tabla 4.

Tabla 4. Índices de diversidad, riqueza y dominancia.

\begin{tabular}{lcc}
\hline Índice & Silvopastoril & Convencional \\
\hline Diversidad de Simpson & 0,6 & 0,17 \\
Diversidad de Shannon & 0,11 & 0,01 \\
Riqueza de Margalef & 9,48 & 6,29 \\
Dominancia de Simpson & 0,37 & 0,63 \\
\hline
\end{tabular}


La diversidad encontrada en el sistema silvopastoril fue mayor para los índices de Simpson y Shannon $(0,60$ y 0,11$)$ frente al sistema convencional $(0,17$ y 0,01 respectivamente), e igualmente para la riqueza específica expresada en el Índice de Margaleff, con 9,48 para el SSP y 6,29 para el SC. Lo anterior se corrobora con la dominancia, expresada en el índice de dominancia de Simpson, con 0,63 en el sistema convencional, por la mayor abundancia de la gramínea Paspalum sp. El sistema silvopastoril muestra una mejor distribución de sus especies, con un índice de dominancia de 0,37.

\section{Cantidady calidad del forraje}

La producción de biomasa comestible, expresada en la Tabla 4, en el periodo seco, fue significativamente inferior $(\mathrm{P}<0,05)$ a la del periodo lluvioso en ambos sistemas. Al comparar la cantidad de materia seca cosechada en cada época, se observó una diferencia $(\mathrm{P}<0,05)$ importante de 2,12 toneladas a favor del sistema silvopastoril. En el periodo de lluvias, la diferencia entre los dos sistemas resultó mucho mayor $(\mathrm{P}<0,05)$, en 3,73 ton de forraje adicional en el SSP. Los resultados de producción anual de biomasa resultaron superiores $(\mathrm{P}<0,05)$ a favor del SSP al obtener 17,82 frente a 11,97 ton de MS ha-1 del sistema convencional basado sólo en gramíneas. Estos resultados difieren de los encontrados en un estudio en bosque húmedo tropical del oriente de Yucatán, México, donde no encontraron diferencia entre la producción de MS de Brachiaria brizantha en monocultivo y asociada a árboles dispersos en el potrero (Itzá-Kinil et al. 2019).

Respecto a la composición nutricional (Tabla 5), ésta se realizó sobre las dos especies más representativas de los dos sistemas. En ellos se pudo comprobar que la gramínea Paspalum sp. contiene mayor nivel de MS (20,83\%), hecho que obedece fundamentalmente a su estructura y característica genética. La arbustiva $T$. diversifolia presentó un nivel de MS de 14,58\% del follaje comestible.

Respecto al contenido proteico de estas especies, las diferencias también fueron muy notorias, con un tenor del 22,8\% de T. diversifolia frente a 15,13\% de Paspalum sp. Estos valores son superiores a los reportados por Gallego et al. (2017), quienes hallaron un contenido proteico no superior 14,7\% en T. diversifolia cultivado en condiciones de altura. Los resultados ratifican el buen aporte proteico de esta arbustiva para la alimentación animal y cobran mayor importancia debido a que en la zona de estudio, los niveles proteicos de las pasturas suele ser bajo, requiriendo siempre aportes adicionales de proteína o nitrógeno para lograr rendimientos satisfactorios de los animales. El contenido de grasa, cuantificado por el porciento de extracto etéreo, fue superior en $T$. diversifolia $(3.92 \%)$ respecto al Paspalum sp., que tuvo un valor de 1,32\%. Estos contenidos se asemejan a con los encontrados por Padilla (2013) y Gálvez et al. (2014), quienes reportan valores de EE de 3,4 y 3.0\% respectivamente en T. diversifolia.

En cuanto a la calidad de los forrajes, se obtuvo un porcentaje de proteína de 22,8 en T. diversifolia, planta destinada al ramoneo (segundo estrato), frente a un 15,13\% de Paspalum sp., grama natural, principal componente de los pastizales convencionales presentes en la zona.

Durante décadas se ha estudiado aquella especie vegetal, pues es una especie que puede llegar a contener hasta un $33 \%$ de proteína en sus hojas, como las especies leguminosas; tiene tenores medios de fósforo $(0,3$ o $0,5 \%)$, además de su capacidad de recuperar escasos nutrientes del suelo, gran volumen radicular y, en general, amplio rango de adaptación (Calle y Murgueitio, 2008). Es una 
Tabla 5. Cantidad y calidad del forraje.

\begin{tabular}{|c|c|c|c|c|}
\hline \multicolumn{5}{|c|}{ Producción de biomasa comestible } \\
\hline Sistema & & $\begin{array}{c}\text { Época seca }(\text { Ton MS } \\
\left.\text { ha }^{-1} \text { periodo }^{-1}\right)\end{array}$ & $\begin{array}{c}\text { Época lluvia (Ton MS } \\
\left.\text { ha }^{-1} \text { periodo }^{-1}\right)\end{array}$ & Producción total año \\
\hline Silvopastoril & & $7,64^{\mathrm{a}}$ & $10,18^{\mathrm{c}}$ & $17,82^{\mathrm{a}}$ \\
\hline Convencional & & $5,52^{\mathrm{b}}$ & $6,45^{\mathrm{d}}$ & $11,97^{\mathrm{b}}$ \\
\hline \multicolumn{5}{|c|}{ Análisis químico proximal (\%) } \\
\hline Especie & MS & $\mathrm{PC}$ & $\mathrm{EE}$ & CEN \\
\hline Paspalum sp & 20,83 & 15,13 & 1,32 & 15,74 \\
\hline Tithonia diversifolia & 14,58 & 22,8 & 3,92 & 17,7 \\
\hline
\end{tabular}

Ton: toneladas; MS: materia seca; ha: hectárea

MS: materia seca; PC: proteína cruda; EE: extracto etéreo; CEN: cenizas.

planta promisoria cuando se utiliza para manipular la ecología microbiana ruminal, reducir la población de metanógenos y protozoos, así como para incrementar la población de bacterias celulolíticas. Además, su utilización permite mitigar las emanaciones de metano a la atmósfera, procedente de la fermentación ruminal; lo que contribuye a reducir el efecto que este gas ejerce como efecto invernadero (Ruiz et al. 2016).

\section{Metabolitos secundarios}

La mayoría de las especies presentan en su follaje compuestos antinutricionales utilizados como defensa para evitar el ataque de bacterias, hongos, virus, ramoneo y estrés ambiental. Contenidos de metabolitos secundarios como saponinas y taninos modifican los consorcios microbianos y la fermentación ruminal, convirtiéndose en aliados naturales para la mitigación de las emisiones de metano (Pérez-Can et al. 2019); lo que no sólo beneficia el ambiente, sino que favorece el desarrollo y rendimiento microbiano $\mathrm{y}$, con ellos, la fermentación en retículo-rumen, que redundará en un mejor desempeño del animal. En general, el contenido de metabolitos secundarios fue mínimo, únicamente se reportó esteroles en niveles bajos. Por consiguiente, se consideran forrajes aptos para el consumo de animales rumiantes.

Caracterización del proceso de regeneración natural

El estudio de la composición florística obtuvo un total de 38 especies entre herbáceas, arbustivas y arbóreas, ubicadas dentro de 25 familias botánicas y 35 géneros, siendo las familias más representativas Fabaceae, Urticaceae y Solanaceae. De las seis categorías de regeneración natural evaluadas, la categoría plantones U1 fue la más representativa con 25 especies, seguida de la categoría brinzal con 16, y fustal con 12 especies. Las categorías con menos especies fueron plantones U2 con 4 especies y, por último, establecidos y latizal con 3 especies cada una, reportadas durante todo el estudio.

Cabe destacar que, a pesar de que en la categoría plantones U1 se encontraron más especies, la clase brinzal fue la que sobresalió por presentar una mayor cantidad de individuos, debido a que el efecto de la selección natural y competencia de la vegetación por la luminosidad dentro de un bosque denso, tanto para la germinación como para el desarrollo de la planta, hacen que pocos individuos logren alcanzar categorías de latizal y fustal (Cárdenas, 2011). 
Abundancia de las especies

Las especies con mayor presencia, con un diámetro a la altura de pecho (DAP) mayor a $10 \mathrm{~cm}$, nos muestra una abundancia general de 135 individuos ha ${ }^{-1}$, siendo las especies más abundantes Cecropia peltata con 46 individuos ha ${ }^{-1}$, Vernonanthura patens con 43 individuos ha ${ }^{-1}$ y Ficus paranensis con 14 individuos ha ${ }^{-1}$.

Dominancias absolutas y frecuencias

De acuerdo con las áreas basales calculadas, se destacan las especies Cecropia peltata y Vernonanthura patens como las especies dominantes del área de estudio de la Granja Maragrícola; así mismo, estas especies presentaron un 13\% de ocurrencias en las subparcelas implementadas. Las poblaciones de los individuos vegetales presentes están expuestas a procesos de adaptación tanto del suelo como del clima, y del manejo implementado; sin embargo, se pueden presentar variaciones a nivel de individuos de una misma especie que pueden brindar diferencias en la producción de biomasa comestible y en otras funciones dentro del agroecosistema (Pérez-Almario et al. 2017).

\section{Caracterización de las especies con potencial forrajero del Sistema Silvopastoril}

En el Tabla 6 se reportan las especies con potencial forrajero, entre herbáceas, arbustivas y arbóreas que se encontraron en el sistema silvopastoril, destacándose las familias Poaceae y Asteraceae. Se observaron 14 especies con potencial forrajero, en las cuales se determinó familia, hábito de crecimiento (herbáceo, arbustivo y arbóreo) y forma de uso. Las especies leñosas con potencial forrajero deben cumplir con varios criterios que es necesario estudiar y profundizar en cada zona de vida, como la cantidad y calidad del follaje, interacciones con los componentes herbáceos y arbustivos, época y altura del aprovechamiento forrajero, y la preferencia de los animales (PérezAlmario et al.2017).

Tabla 6. Flora con potencial forrajero presente en el Sistema Silvopastoril.

\begin{tabular}{|c|c|c|c|}
\hline \multicolumn{4}{|c|}{ HERBÁCEAS } \\
\hline NOMBRE COMÚN & NOMBRE CIENTIFICO & FAMILIA & USO \\
\hline Pasto brachiaria & Brachiaria humidicola & Poaceae & Pastoreo \\
\hline Campanilla & Clitoria ternatea $\mathrm{L}$. & Fabaceae & Banco de proteína, pastoreo \\
\hline Santa Elena & Cyperus odoratus L. & Cyperaceae & Pastoreo \\
\hline Pangola & Digitaria decumbens & Poaceae & Corte, pastoreo, ensilaje \\
\hline Kudzu tropical & Pueraria phaseoloides & Fabaceae & Pastoreo, banco de proteína \\
\hline Grama natural & Paspalum sp. & Poaceae & Pastoreo \\
\hline \multicolumn{4}{|c|}{ ARBUSTIVAS } \\
\hline Chilca blanca & Baccharis dracunculifolia & Asteraceae & Pastoreo \\
\hline Botón de oro & Tithonia diversifolia & Asteraceae & Corte, ramoneo \\
\hline Malvilla & Pavonia paniculata & Malvaceae & Pastoreo \\
\hline Papaya & Carica papaya & Caricaceae & Fruto, alimento animal \\
\hline \multicolumn{4}{|c|}{ ARBÓREAS } \\
\hline Calabazo & Crescentia cujete & Bignoniaceae & Sombra, alimento animal \\
\hline Guanábana & Annona muricata & Annonaceae & Forrajera \\
\hline Limón & Citrus limón & Rutaceae & Fruto, alimento animal \\
\hline
\end{tabular}




\section{CONCLUSIONES}

La actividad de dragado para la construcción de las piscinas camaroneras en la Granja Maragrícola generó un deterioro evidente del perfil cultivable del suelo, afectando su diversidad de flora y fauna, debido al excesivo pisoteo. Los sistemas silvopastoriles mejoran las propiedades físicas del suelo, las cuales estaban afectadas debido a la actividad agrícola a la que estaban destinadas, pues los SSP contribuyen de manera directa con la adición de materia orgánica y hojarasca, pero también estos árboles, debido a su crecimiento radicular, generan una resistencia mecánica del suelo a la penetración de las raíces, mejorando no sólo las propiedades químicas si no también las físicas, lo que demuestra que los SSP son una herramienta eficaz para recuperar suelos degradados por las actividades agropecuarias.

El proceso de regeneración natural permitió una recuperación paulatina de las condiciones favorables del suelo que posibilitan el surgimiento de un nuevo componente vegetal aprovechable para la alimentación animal.

La concentración del fósforo y potasio presentó un incremento considerable dentro del sistema silvopastoril, como resultado de la hojarasca al suelo y su mineralización.

Los sistemas silvopastoriles incrementaron significativamente la producción de biomasa comestible y el contenido proteico de la oferta forrajera debido a la oferta adicional de follaje arbóreo y arbustivo con mayores porcentajes de Nitrógeno, condición específica de los SSP.

Se logró identificar 14 especies con potencial forrajero en el área de estudio, producto del proceso de regeneración natural, importantes por su mejora en la cantidad y calidad de la dieta a través del año, además de brindar sistemas más complejos y estables en los sistemas ganaderos del bosque húmedo.

\section{LITERATURA CITADA}

ANDERSON, J.M.; INGRAM, J.S.I. Eds. Tropical soil biology and fertility. A handbook of methods. 2nd ed. Wallingford, United Kingdom: CAB International, 1993.221p.

A.O.A.C. (Official Methods of Analysis. Ass. Off.). Agricultural Chemist. 16thed. Washington, D.C. USA, 1995. pp 2201-3301.

CALLE, Z; MURGUEITIO, E. El botón de oro: arbusto de gran utilidadpara sistemas ganaderos de tierra caliente y de montaña, Centro para la Investigación en Sistemas Sostenibles de Producción Agropecuaria (Cipav). Ganadería del futuro: investigación para el desarrollo. Fundación CIPAV. 2008. p. 55. Disponible en internet: http://nutriciondebovinos.com.ar/MD_upload/nutriciondebovinos_com_ar/Archivos/ File/Boton_de_Oro_y_Ganaderia.pdf.

CÁRDENAS, P. ..l"Regeneración natural de Cedrelinga catenaeformis Ducke en bosque intervenido de la comunidad nativa Catungo Quimpiri - Río Tambo - Junín”. Tesis de Especialidad de Ingeniería Forestal, Facultad de Ciencia Agrarias, Universidad Nacional del Centro del Perú. 2011. 26p.

GALLEGO, L; MAHECHA, L; ANGULO, J. Calidad nutricional de Tithonia diversifolia Hemsl. A Gray bajo tres sistemas de siembra en el trópico alto. Agronomía Mesoamericana, 28(1), 2017. 213p. https://doi.org/10.15517/am.v28i1.21671

GÁlVEZ-CERÓN, A; LAGOS-ROSERO, Y; ARMERO-HERNÁNDEZ, C. Caracterización del 
componente herbáceo y arbustivo de un sistema silvopastoril por regeneración natural en una zona de bosque seco tropical (bs-T) del departamento de Nariño. Revista Investigación Pecuarias. REVIP. Universidad de Nariño, Pasto-Nariño. 3(1): 57-72. Noviembre 2014.

GÁLVEZ-CERÓN A. Dieta del rebeco en el Pirineo oriental: efectos del ganado doméstico y de los parásitos. Tesis de grado para obtar al título de Doctorado en Producción Animal. Barcelona, España, Universidad Autónoma de Barcelona, 2015. 183 p.

GÁlVEZ-CERON, A; BOLAÑOS-BOLAÑOS, M; GÓMEZ-DAZA, Y. Valoración de sistemas convencional y silvopastoril multiestrato en bosque muy seco tropical. Revista Agro Sur 43(3): 41-48, 2018. DOI:10.4206/agrosur.2018.v43n3-05.

GARCÍA, J. Crecimiento y calidad de gramíneas forrajeras en La Estanzuela. (en línea). Montevideo, Uruguay, INIA. 35 p. (Serie Técnica no. 133). 2003. Consultado 1 ago. 2019. Disponible en http://www.ainfo.inia.uy/digital/bitstream/item/2878/1/15630191107 142500.pdf

GAVIRIA X, RIVERA J.E, BARAHONA R. Calidad nutricional y fraccionamiento de carbohidratos y proteína en los componentes forrajeros de un sistema silvopastoril intensivo. Pastos y Forrajes;38(2):194201. Sep. 2015.

HERNÁNDEZ-VIGOA, G; CABRERA-DÁVILA, G; IZQUIERDO-BRITO, I; SOCARRÁS-RIVERO, A.A; HERNÁNDEZ-MARTÍNEZ, L; SÁNCHEZ-RENDÓN, J.A. Indicadores edáficos después de la conversión de un pastizal a sistemas Agroecológicos. Pastos y Forrajes, Vol. 41, No. 1, pp 3-12. enero-marzo, 2018.

HOLDRIDGE, L. Ecología basada en zonas de vida. Instituto Interamericano de cooperación para la agricultura (IICA). Serie de libros y materiales educativos No. 34. 1978.

ITZÁ-KINIL, W; KUMUL-CHIMAL, F; COUOH-PUC, A.J; LUNA-MENDICUTI, A.A; PINEDADOPORTO, A; CASTILLO-SÁNCHEZ, L.E; ALVARADO-CANCHÉ, A.R; CAMPOS-NAVARRETE, M.J; CHAY-CANUL, A.J; PIÑEIRO-VÁZQUEZ, A.T; CANUL-SOLIS, J.R. Producción Forrajera de Brachiaria brizantha bajo Monocultivo y Sistema Silvopastoril. En: Agroecosistemas tropicales: conservación de recursos naturales y seguridad alimentaria. CETZAL-IX, W.; CASANOVA-LUGO, F.; CHAY-CANUL, A.J.; JESÚS F. MARTÍNEZ-PUC, J.F. (Eds). México: Tecnológico Nacional de México, Instituto Tecnológico de Chiná, Instituto Tecnológico de la Zona Maya. 422-426p. 2019.

LINARES, J. Evaluación de algunas propiedades del suelo como indicadoras de sostenibilidad para pastoreo rotacional en silvopastoreo en un Endoacuept del Valle medio del río Sinú Colombia. Tesis de grado para optar al titulo de Magister en Ciencias Agrarias. Palmira, Valle del Cauca. Universidad Nacional de Colombia, sede Palmira. Facultad de Agronomía. 2006. 126p.

MARTÍNEZ, J. Producción y descomposición de hojarasca en sistemas silvopastoriles de estratos múltiples y su efecto sobre propiedades bioorgánicas del suelo en el valle medio del Río Sinú. Tesis para optar al título de Doctor en Ciencias Agrarias - Área Agraria. Director: Ph.D Nelson Walter Osorio Vega. Medellín, Universidad Nacional de Colombia, Facultad de Ciencias Agrarias, 2013. 161p.

MARTÍNEZ, J; CAJAS, YS; LEÓN, JD; OSORIO, NW. Silvopastoral Systems Enhance Soil Quality in Grasslands of Colombia. Applied and Environmental Soil Science 1-8. Doi: 10.1155/2014/359736, junio, 2014. 
MAYA-MARTÍNEZ, A.; DEL ÁNGEL-PÉREZ, A.L.; HERNÁNDEZ-GARCÍA, G.; CANALES-CRUZ, R.; NATAREN-VELÁZQUEZ, J.; ROSADO-CALDERÓN, A.T. Módulos Agroforestales de Producción Diversificada e Intensiva en Selva Húmeda de México. En: Agroecosistemas tropicales: conservación de recursos naturales y seguridad alimentaria. CETZAL-IX, W.; CASANOVA-LUGO, F.; CHAY-CANUL, A.J.; JESÚS F. Campeche, Chiná, Mexico. 533p. Junio, 2019.

MELO, O; VARGAS, R. Evaluación ecológica y silvicultural de ecosistemas boscosos. Universidad del Tolima, CRQ, CARDER, CORPOCALDAS, CORTOLIMA. Ibague. 235p. 2003.

MORENO, C. Métodos para medir la biodiversidad: M\&T- Manuales y tesis, SEA. Zaragoza, España: CYTED-ORCYT UNESCO'-SEA. 235 p. 2001.

OSPINA, S; RUSCH, G; EASDALE, T; FINEGAN, B; CASANOVES, F; IBRAHIM, M. Community aggregated traits disclose functional responses to seasonal resource fluctuations and spatial heterogeneity. Journal of Vegetation Science, 27 (6). 2016.

PADILLA, M. Evaluación de la producción cuyícola bajo arreglos silvopastoriles con botón de oro (Tithonia diversifolia), acacia de la pradera (Senegalia angustissima), reventador (Clibadium sp), Guatemala (Tripsacum andersonii) e imperial (Axonopus scoparius), en clima medio del departamento de Nariño: Tesis para optar al titulo de Magister en Ciencias Agrarias, Universidad de Nariño, Pasto, Colombia. Programa de Zootecnia, 2013.106p.

PÉREZ, A; MATÍAS, C; GONZÁLEZ, Y; ALONSO, O. Producción de semillas de gramíneas y leguminosas tropicales. En: Recursos forrajeros herbáceos y arbóreos. Guatemala: Universidad de San Carlos, EEPF Indio Hatuey.p. 103-128. 2006.

PÉREZ-ALMARIO, N; OSPINA, S; MORA, J; CRIOLLO, D; MEDINA, E. Atributos funcionales a considerar en la selección de especies leñosas para el diseño de sistemas silvopastoriles en zonas secas. Manizales, Colombia. IX congreso internacional sistemas silvopastoriles. Manizales, Colombia, CIPAV, capítulo 1, 14-21. ISBN 978-958-9386-78-1.2017.

PÉREZ-CAN, G.E.; UC-ZAPATA, W.G.; UICAB-CHIM, D.E.; SANGUINÉS-GARCÍA, R.; AGUILARURQUIZO, E.; CHAY-CANUL, A.J.; CASANOVA-LUGO, F.; CANUL-SOLIS, J.R.; G. JIMÉNEZFERRER, G.; J.A. ALAYON-GAMBOA, J.A.; A.T. PIÑEIRO-VÁZQUEZ, A.T. Árboles y Arbustos Tropicales y su Contribución en la Alimentación de Rumiantes. En: Agroecosistemas tropicales: conservación de recursos naturales y seguridad alimentaria. CETZAL-IX, W.; CASANOVA-LUGO, F.; CHAY-CANUL, A.J.; JESÚS F. MARTÍNEZ-PUC, J.F. (Eds). México: Tecnológico Nacional de México, Instituto Tecnológico de Chiná, Instituto Tecnológico de la Zona Maya. 408-415p. 2019.

PERLA, C; TORRES, J. Caracterización de la vegetación forestal, usos y diversidad de especies de la vegetación forestal en la Reserva Privada Escameca Grande, San Juan del Sur, Rivas, Managua, Nicaragua. Trabajo de Diplomado, Facultad de Recursos Naturales y del Ambiente. Universidad Nacional Agraria. Nicaragua. 27p. 2008.

PRIANO, ME; FUSÉ, VS; MESTELAN, S; BERKOVIC, AM; GUZMÁN, SA; GRATTON, R; JULIARENA, MP. Afforested sites in a temperate grassland region: influence on soil properties and methane uptake. Agroforestry Systems 92(2):311-320. Doi: https:// doi.org/10.1007/s10457-017-0104-7. 2017. 
REINA, A; MENESES, E. Cuantificación de la macrofauna edáfica en un sistema silvopastoril y uno convencional en bosque seco. Tesis de grado para optar al título de Zootecnista. Facultad de Ciencias Pecuarias. Universidad de Nariño. Junio de 2015.

RICO, G. Colombia: la ganadería extensiva está acabando con los bosques. Mongabay Latam. 17 de enero de 2017. Disponible en internet: https://es.mongabay.com/2017/01/colombia-ganaderia-deforestacion/

RUIZ, T.E; ALONSO, J; FEBLES, G.J; GALINDO, J.L; LOURDES L; SAVÓN, L.L; CHONGO, B.B. Universidad de Colima, Centro Universitario de Investigaciones Sociales. Avances en Investigacion A gropecuaria (Vol. 20, Issue 3). 2016 . Disponible en internet: https://go.gale.com/ps/anonymous? id=GALE\%7CA500823720\&sid=googleScholar\&v=2.1\&it=r\&linkacc $\underline{\text { ess }=\text { abs } \& \text { issn }=01887890 \& p=I F M E \& s w=w}$.

STEFANO, A; JACOBSON, MG. Soil carbon sequestration in agroforestry systems: a meta-analysis. Agroforest Syst 92(2):285-299. Doi: https://doi.org/10.1007/s10457-017-0147-9. 2017.

VALLEJO, VE; ROLDÁN, F; DICK, RP. Soil enzymatic activities and microbial biomass in an integrated agroforestry chronosequence compared to monoculture and a native forest in Colombia. Biology and Fertility of Soils 46(6):577-587. Doi: https://doi.org/10.1007/s00374-010-0466-8. 2012.

VILLARREAL, H; ÁLVAREZ, M; CÓRDOBA, S; ESCOBAR, F; FAGUA, G; GAST, F; MENDOZA, H; OSPINA, M; UMAÑA, M. Manual de métodos para el desarrollo de inventarios de biodiversidad. Instituto de Investigación de Recursos Biológicos Alexander von Humboldt Programa Inventarios de Biodiversidad Segunda edición. Bogotá, Colombia. 236 p. 2006. 